Pacific Journal of Mathematics

ON DOMINATED EXTENSIONS IN LINEAR SUBSPACES OF

Alfsen and B. Hirsberg 


\title{
ON DOMINATED EXTENSIONS IN LINEAR SUBSPACES OF $\mathscr{C}_{c}(X)$
}

\author{
E. M. Alfsen AND B. Hirsberg
}

The main result is the following: Given a closed linear subspace $A$ of $\mathscr{C}_{\mathrm{C}}(X)$ where $X$ is compact Hausdorff and $A$ contains constants and separates points, and let $F$ be a compact subset of the Choquet boundary $\partial_{A} X$ with the property that the restriction to $F$ of every $A$-orthogonal boundary measure remains orthogonal. If $\left.a_{0} \in A\right|_{F}$ and $a_{0} \leqq\left.\Psi\right|_{F}$ for some strictly positive $A$-superharmonic function $\Psi$, then $a_{0}$ can be extended to a function $a \in A$ such that $a \leqq \Psi$ on all of $X$. It is shown how this result is related to various known dominated extension-and peak set-theorems for linear spaces and algebras. In particular, it is shown how it generalizes the Bishop-Rudin-Carleson Theorem.

The aim of this paper is to study extensions within a given linear subspace $A$ of $\mathscr{C}_{C}(X)$ of functions defined on a compact subset of the Choquet boundary $\partial_{A} X$, in such a way that the extended function remains dominated by a given $A$-superharmonic function $\Psi$. (Precise definitions follow). Our main result is the possibility of such extensions for all functions in $\left.A\right|_{F}$ provided $F$ satisfies the crucial requirement that the restriction to $F$ of every orthogonal boundary measure shall remain orthogonal (Theorem 4.5). Taking $\Psi \equiv 1$ in this theorem we obtain that $F$ has the norm preserving extension property (Corollary 4.6). This was first stated by Björk [5] for a real linear subspace $A$ of $\mathscr{C}_{R}(X)$ and for a metrizable $X$. A geometric proof of the latter result was given by Bai Andersen [3]. In fact, he derived it from a general property of split faces of compact convex sets, which he proved by a modification of an inductive construction devised by Pelczynski for the study of simultaneous extensions within $\mathscr{C}_{R}(X)$ [12]. Our treatment of the more general extension property proceeds along the same lines as Bai Andersen's work. It depends strongly upon the geometry of the state space of $A$, and Bai Andersen's construction is applied at an essential point in the proof. Note however, that this is no mere translation of real arguments. The presence of complex orthogonal measures seems to present a basically new situation. Applying arguments similar to those indicated above, we obtain a general peak set-and peak point criterion (Theorem 5.4 and Corollary 5.5) of which the latter has been proved for real spaces by Björk [6]. In $\S 6$ (Theorem 6.1) it is shown how the Bishop-Rudin-Carleson Theorem follows from the general extension theorem mentioned above. In $\S 7$ we assume that $A$ is a sup-norm algebra over $X$ and study the inter- 
relationship between our conditions on $F$ and a condition introduced by Gamelin and Glicksberg [9], [10]. Finally we should like point out that some related investigations have been carried out recently by Brièm [7]. However, his methods are rather different. The geometry of the state space is not invoked, but instead he applies in an essential way a measurable selection theorem of Rao [14].

We want to thank Bai Andersen for many stimulating discussions of the problems of the present paper. Also we are indebted to A. M. Davie for the counterexample at the end of $\S 7$.

1. Preliminaries and notation. In this note $X$ shall denote a compact Hausdorff space and $A$ a closed, linear subspace of $\mathscr{C}_{c}(X)$, which separates the points of $X$ and contains the constant functions.

The state space of $A$, i.e.

$$
S=\left\{p \in A^{*} \mid p(1)=\|p\|=1\right\},
$$

is convex and compact in the $w^{*}$-topology. Since $A$ separates the points of $X$, we have a homeomorphic embedding $\Phi$ of $X$ into $S$, defined by

$$
\Phi(x)(a)=a(x), \quad \text { all } \quad a \in A .
$$

Similary we have an embedding $\Psi$ of $A$ into the space $A_{C}(S)$ of all complex valued $w^{*}$-continuous affine functions on $S$; namely

$$
\Psi(a)(p)=p(a), \text { all } p \in S .
$$

By taking real parts of the functions $\Psi(a)$ we obtain the linear space of those real valued $w^{*}$-continuous affine functions on $S$, which can be extended to real valued $w^{*}$-continuous linear functionals on $A^{*}$, and this space $A_{R}\left(S, A^{*}\right)$ is dense in the space $A_{R}(S)$ of all real valued affine $w^{*}$-continuous functions on $S$, [1, Cor. I. 1.5].

We shall denote by $M(X)$, resp. $M(S)$, the Banach space of all complex Radon measures on $X$, resp. $S$; by $M^{+}(X)$ resp. $M^{+}(S)$ the cone of positive (real) measures, and by $M_{1}^{+}(X)$ resp. $M_{1}^{+}(S)$ the $w^{*}$-compact convex set of probability measures. The set of extreme points of $S$ will be denoted by $\partial_{e} S$, and the Choquet boundary of $X$ with respect to $A$ is defined as the set

$$
\partial_{A} X=\left\{x \in X \mid \Phi(x) \in \partial_{e} S\right\} .
$$

From [13, p. 38] it follows that $\partial_{e} S \subset \Phi(X)$ so that $\Phi$ maps $\partial_{A} X$ homeomorphically onto $\partial_{e} S$.

A measure $\mu \in M(S)$ is said to be a boundary measure on $S$ if the total variation $|\mu|$ is a maximal measure in Choquet's ordering of positive measures [1, ch. I, §3], [13, p. 24]. A boundary measure 
is supported by $\overline{\partial_{e} S}$ [1, Prop. I. 4.6]. For a metrizable $X$ (and $S$ ) a measure $\mu \in M(S)$ is a boundary measure if and only if $|\mu|\left(S \mid \partial_{e} S\right)=0$. We shall denote by $M\left(\partial_{e} S\right)$ the set of boundary measures on $S$ (abuse of language). Observe that if $\mu \in M\left(\partial_{e} S\right)$, then the real and imaginary parts of $\mu$ are both boundary measures. The set of boundary measures on $X$ is defined by

$$
M\left(\partial_{A} X\right)=\left\{\mu \in M(X) \mid \Phi \mu \in M\left(\partial_{e} S\right)\right\},
$$

where $\Phi \mu$ denotes the transport of the measure $\mu$ on $X$ to a measure on $S$. For a metrizable $X$ a measure $\mu$ on $X$ belongs to $M\left(\partial_{A} X\right)$ if and only if $|\mu|\left(X \mid \partial_{A} X\right)=0$.

For every $\mu \in M_{1}^{+}(S)$ we shall use the symbol $r(\mu)$ to denote the barycenter of $\mu$, i.e., the unique point in $S$ such that $a(r(\mu))=\mu(a)$ for all $a \in A_{R}(S)$. The Choquet-Bishop de Leeuw Theorem states that each point in $S$ is the barycenter of a maximal (boundary) probability measure [1, Th. I. 4.8]. Accordingly we shall denote by $M_{p}^{+}\left(\partial_{e} S\right)$ the nonempty set of maximal (boundary) probability measures on $S$ with barycenter $p \in S$. For $x \in X$ we define $M_{x}^{+}\left(\partial_{A} X\right)$ to be the set of all $\mu \in M_{1}^{+}(X)$ such that $\Phi \mu \in M_{\Phi(x)}^{+}\left(\partial_{e} S\right)$. Equivalently, $M_{x}^{+}\left(\partial_{A} X\right)$ consists of all $\mu \in M_{1}^{+}\left(\partial_{A} X\right)$ such that

$$
a(x)=\int a d \mu \text { all } a \in A,
$$

i.e., $\mu$ represents $x$ with respect to $A$. Also we denote by $M_{x}^{+}(X)$ the set of probability measures on all of $X$ which represents $x$ in this way. Similary we denote by $M_{p}^{+}(S)$ the set of probability measures on $S$ with barycenter $p$. The annihilator of $A$ in $M(X)$ is the set

$$
A^{\perp}=\{\mu \in M(X) \mid \mu(a)=0 \text { all } a \in A\} .
$$

Finally we shall use the symbol $\mathscr{B}(X)$ to denote the class of all complex valued bounded Borel functions on $X$.

2. A dominated extension theorem. We start by proving a general dominated extension theorem, which may be of some independant interest. In this connection we give the following:

Definition 2.1. $\mathscr{A}$ is the class of all $f \in \mathscr{B}(X)$ such that

$$
\mu(f)=0 \text { all } \mu \in A^{\perp} \text {. }
$$

Clearly $A \subset \mathscr{A}$.

THEOREM 2.2. Let $F$ be a closed subset of $X$ for which $\left.A\right|_{F}=$ $\left\{\left.a\right|_{F} \mid a \in A\right\}$ is closed in $\mathscr{C}_{\boldsymbol{C}}(F)$; let $\left.a_{0} \in A\right|_{F}$ and let $\varphi: X \rightarrow \boldsymbol{R}^{+} \cup\{\infty\}$ be 
a strictly positive l.s.c. function such that $\left|a_{0}(x)\right|<\varphi(x)$ for all $x \in F_{\text {. }}$.

Now, if there exists a function $\bar{a}_{0} \in \mathscr{A}$ such that

$$
\left.\bar{a}_{0}\right|_{F}=a_{0}, \quad\left|\bar{a}_{0}(x)\right|<\varphi(x) \text { all } x \in X
$$

then there exists a function in $A$ with the same properties.

Proof. Without lack of generality we can assume that $\varphi$ is a. bounded function with values in $\boldsymbol{R}^{+}$, and we assume for contradiction that

$$
\left.a_{0} \notin G\right|_{F^{\prime}}=\left\{\left.a\right|_{F} \mid a \in G\right\},
$$

where

$$
G=\{a \in A|| a(x) \mid<\varphi(x)\} .
$$

Since $\varphi$ is l.s.c., $G$ is an open subset of $A$. Since $\left.A\right|_{F}$ is closed in $\mathscr{C}_{C}(F)$, we may apply the Open Mapping Theorem to the restriction map $R_{F}:\left.A \rightarrow A\right|_{F}$. Hence $\left.G\right|_{F}$ is an open subset of $\left.A\right|_{F}$. Furthermore $\left.G\right|_{F}$ is convex and circled. By the Hahn-Banach Theorem we can find a measure $\nu \in M(X)$ with supp $\nu \subset F$ such that

$$
\nu\left(a_{0}\right) \geqq 1 \geqq\left|\nu\left(b_{0}\right)\right| \text { all }\left.b_{0} \in G\right|_{F} .
$$

Now we consider $\mathscr{C}_{\boldsymbol{C}}(X)$ equipped with the norm

$$
\|f\|_{\varphi}=\sup \left\{\frac{|f(x)|}{\varphi(x)} \mid x \in X\right\},
$$

and observe that this norm is topologically equivalent with the customary, uniform norm. The dual of $\left(\mathscr{C}_{c}(X),\|-\|_{\varphi}\right)$ is seen to be $M(X)$ equipped with the norm $\|\mu\|_{\varphi}=\|\varphi \cdot \mu\|$, where $(\varphi \cdot \mu)(f)=\mu(\varphi f)$ for all $f \in \mathscr{C}_{c}(X)$.

It follows from (2.5) that the linear functional $\xi$ on $\left(A,\|-\|_{\varphi}\right)$, defined by

$$
\xi(a)=\nu\left(R_{F} a\right) \text { all } a \in A,
$$

is bounded with norm $\|\xi\|_{\varphi} \leqq 1$. Now we extend $\xi$ with preservation of $\varphi$-norm to a bounded linear functional on $\left(\mathscr{C}_{\boldsymbol{c}}(X),\|-\|_{\varphi}\right)$. This: gives a measure $\mu \in M(X)$, such that

$$
\xi(a)=\mu(a) \text { all } a \in A,\|\varphi \cdot \mu\|=\|\xi\|_{\varphi} \leqq 1 .
$$

It follows from (2.2) and (2.8) that

$$
\left|\mu\left(\bar{a}_{0}\right)\right|=\left|(\varphi \cdot \mu)\left(\phi^{-1} \bar{a}_{0}\right)\right|<1 .
$$

From (2.7) and (2.8) it follows that $\mu-\nu \in A^{\perp}$, and since $\bar{a}_{0} \in \mathscr{A}$ we shall have 


$$
\left|\int_{X} \bar{a}_{0} d \mu\right|=\left|\int_{X} \bar{a}_{0} d \nu\right|=\int_{F} a_{0} d \nu \geqq 1
$$

This contradicts (2.9) and the proof is complete.

3. Applications of the geometry of the state space. We shall consider compact subsets $F$ of $\partial_{A} X$ satisfying one or the other of the following two requirements:

$$
\begin{aligned}
& \left.\mu \in M\left(\partial_{A} X\right) \cap A^{\perp} \Rightarrow \mu\right|_{F} \in A^{\perp} \\
& \mu \in M\left(\partial_{A} X\right) \cap A^{\perp} \Rightarrow \mu(F)=0 .
\end{aligned}
$$

We assume first (A.1). We also agree to write $S_{F}=\overline{c o}(\Phi(F))$, and we observe that there is a canonical embedding $\Psi_{F}$ of $\left.A\right|_{F}$ into $A_{C}\left(S_{F}\right)$, defined by

$$
\Psi_{F}\left(a_{0}\right)(p)=p(a), \quad \text { all } \quad p \in S_{F}
$$

where $a \in A ;\left.\alpha\right|_{F}=a_{0}$. In fact, it follows by the integral form of the Krein-Milman Theorem that $p$ can be expressed as the barycenter of a probability measure on $\Phi(F)$, and hence that the particular choice of $a$ is immaterial.

For every $\left.a_{0} \in A\right|_{F}$ we define

$$
\bar{a}_{0}(x)=\int_{F} a_{0} d \mu_{x}, \quad x \in X, \mu_{x} \in M_{x}^{+}\left(\partial_{A} X\right),
$$

and

$$
\overline{\bar{a}}_{\nu}(p)=\int_{S_{F}} \Psi_{F}\left(a_{0}\right) d \mu_{p}, \quad p \in S, \mu_{p} \in M_{p}^{+}\left(\partial_{e} S\right),
$$

and we note that these definitions are legitimate by virtue of (A.1). We also note that $\mu_{p}\left(S_{F}\right)=\mu_{p}\left(\Phi\left(F^{\prime}\right)\right)$ for all $p \in S$ and $\mu_{p} \in M_{p}^{+}\left(\partial_{e} S\right)$ [3, Lem. 1].

Clearly $\bar{a}_{0}$ is an extension of $a_{0}$ to a function defined on all of $X$; and if we think of $\Phi$ as an imbedding of $X$ into $S$, then $\overline{\bar{a}}_{0}$ will in turn be an extension of $\bar{a}_{0}$ to a function defined on all $S$. More specifically, for every $\mu_{x} \in M_{x}^{+}\left(\partial_{A} X\right)$ the transported measure $\Phi \mu_{x}$ is in $M_{\Phi(x)}^{+}\left(\partial_{e} S\right)$ and so

$$
\overline{\bar{a}}_{0}(\Phi(X))=\int_{S_{F}} \Psi_{F}\left(a_{0}\right) d\left(\Phi \mu_{x}\right)=\int_{F} \Psi_{F}\left(a_{0}\right) \circ \Phi d \mu_{x}=\int_{F} a_{0} d \mu_{x},
$$

which entails

$$
\overline{\bar{a}}_{\jmath} \circ \Phi=\bar{a}_{0} .
$$

Lemma 3.1. If $F$ satisfies (A.1) and $\left.a_{0} \in A\right|_{F}$, then $\bar{a}_{0} \in \mathscr{A}$. 
Proof. Let $\lambda=\left\|a_{0}\right\|_{F}$ and define

$$
a_{1}=\operatorname{Re} \Psi_{F}\left(a_{0}\right)+\lambda, \quad a_{2}=\operatorname{Im} \Psi_{F}\left(a_{0}\right)+\lambda .
$$

Then $a_{1}, a_{2} \in A_{R}\left(S_{F}\right)^{+}$and for any $p \in S$ and $\mu_{p} \in M_{p}^{+}\left(\partial_{e} S\right)$

$$
\overline{\bar{a}}_{0}(p)=\int_{S_{F}} \Psi_{F}\left(a_{0}\right) d \mu_{p}=\int_{S_{F}} a_{1} d \mu_{p}+i \int_{S_{F}} a_{2} d \mu_{p}-\lambda \mu_{p}\left(S_{F}\right)-i \lambda \mu_{p}\left(S_{F}\right) .
$$

At this point we shall appeal to the geometric theory of compact convex sets. We recall that a face $Q$ of $S$ is said to be split if the complementary face $Q^{\prime}$ (=the union of all faces disjoint from $Q$ ) is convex (hence a face) and every element of $S$ can be expressed by a unique convex combination of an element of $Q$ and an element of $Q^{\prime}$. It is known ([1, Th. II. 6.12], [1, Th. II. 6.18], see also [2, Th. 3.5]) that for a closed face $Q$ of $S$ the following statements are equivalent:

(i ) If a real measure $\mu \in M\left(\partial_{e} S\right)$ annihilates all continuous affine functions, then $\left.\mu\right|_{Q}$ has the same property.

(ii) $Q$ is a split face.

(iii) The u.s.c. concave upper envelope $\widehat{b \chi_{Q}}$ of the function $b \chi_{Q}$ which is equal to $b$ on $Q$ and 0 on $S \backslash Q$, is affine for every $b \in A_{R}(Q)^{+}$.

It follows from the requirement (A.1) that $S_{F}$ is a split face of $S$, and hence that

$$
\overline{\bar{a}}_{0}(p)=\widehat{a_{1} \chi_{S_{F}}}(p)+i \widehat{a_{2} \widehat{\chi}_{S_{F}}}(p)-\lambda \hat{\chi}_{S_{F}}(p)-i \lambda \hat{\chi}_{S_{F}}(p),
$$

where all the functions on the right hand side are u.s.c. and affine. In particular $\overline{\bar{a}}_{0}$ is a Borel function, and it follows from (3.4) that $\bar{a}_{0}$. is a Borel function as well. Since the barycentric calculus applies to real valued u.s.c. affine functions on $S$ [1, Cor. I 1.4], we shall have:

$$
\overline{\bar{a}}_{0}(p)=\int_{S} \overline{\bar{a}}_{0} d \mu_{p}, \quad p \in S, \mu_{p} \in M_{p}^{+}(S) .
$$

Let $\mu \in A^{\perp}$ be arbitrary and decompose

$$
\mu=\sum_{i=1}^{4} a_{i} \mu_{i},
$$

where $\alpha_{1} \in \boldsymbol{R}^{+}, \alpha_{2} \in-\boldsymbol{R}^{+}, \alpha_{3} \in i \boldsymbol{R}^{+}, \alpha_{4} \in(-i) \boldsymbol{R}^{+}$and $\mu_{i} \in M_{1}^{+}(X)$ for $i=$ $1,2,3,4$. Let $p_{i} \in S$ be the barycenter $\Phi \mu_{i}$ and let $\sigma_{i} \in M_{p_{i}}^{+}\left(\partial_{e} S\right)$ for $i=1,2,3,4$.

Since $\overline{\partial_{e} S} \subseteq \Phi(X)$ we can transport $\sigma_{i}$ back to $X$ by the map $\Phi^{-1}$, and it follows that the measures $\mu_{i}-\Phi^{-1} \sigma_{i}$ are (real) orthogonal measures for $i=1,2,3,4$.

Writing

$$
\tau=\sum_{i=1}^{4} \alpha_{i} \Phi^{-1} \sigma_{i}
$$


we obtain $\tau \in M\left(\partial_{A} X\right)$ and $\mu-\tau \in A^{\perp}$. In fact for every $a \in A$,

$$
\int a d(\mu-\tau)=\int_{S} \Psi(a) d(\Phi(\mu-\tau))=\sum_{i=1}^{4} \alpha_{i} \int_{S} \Psi(\alpha) d\left(\Phi \mu_{i}-\sigma_{i}\right)=0 .
$$

Since $\mu \in A^{\perp}$, we shall also have $\tau \in A^{\perp}$ and then $\left.\tau\right|_{F} \in A^{\perp}$ by virtue of (A.1). Hence by (3.3), (3.4), (3.5):

$$
\begin{aligned}
\int_{X} \bar{a}_{0} d \mu & =\int_{X} \overline{\bar{a}} \circ \Phi d \mu=\int_{S} \overline{\bar{a}}_{0} d(\Phi \mu)=\sum_{i=1}^{4} \alpha_{i} \int_{S} \overline{\bar{a}}_{0} d\left(\Phi \mu_{i}\right) \\
& =\sum_{i=1}^{4} \alpha_{i} \overline{\bar{a}}_{j}\left(p_{i}\right)=\sum_{i=1}^{4} \alpha_{i} \int_{S_{F}} \Psi_{F}\left(a_{0}\right) d \sigma_{i}=\int_{S_{F}} \Psi_{F}\left(a_{0}\right) d(\Phi \tau) \\
& =\int_{F} a_{0} d \tau=0 .
\end{aligned}
$$

Hence $\bar{a}_{0} \in \mathscr{A}$, and the proof is complete.

We next turn to the less restrictive requirement (A.2). It follows by a slight modification of the proof of [1, Th. II. 6.12], that the requirement (A.2) implies that $S_{F}$ is a parallel face of $S$ and hence that the function $\hat{\chi}_{S_{F}}$ is affine [15, Th. 12].

For every $x \in X$ we define

$$
\bar{\chi}_{F}(x)=\int_{F} 1 d \mu_{x}, \quad \mu_{x} \in M_{x}^{+}\left(\partial_{A} X\right)
$$

and we note that this definition is legitimate by virtue of (A.2). For $x \in X$ and $\mu_{x} \in M_{x}^{+}\left(\partial_{A} X\right)$ we shall have:

$$
\hat{\chi}_{S_{F}}(\Phi(x))=\int_{S_{F}} 1 d\left(\Phi \mu_{x}\right)=\int_{F} 1 d \mu_{x}=\bar{\chi}_{F}(x)
$$

which entails

$$
\hat{\chi}_{S_{F}} \circ \Phi=\bar{\chi}_{F} .
$$

Applying (3.8) and proceeding as in the proof of Lemma 3.1, we can prove

Lemma 3.2. If $F$ satisfies (A.2), then $\bar{\chi}_{F} \in \mathscr{A}$

4. Extensions dominated by $A$-superharmonic functions. We now proceed to the main theorem, but first we give some definitions.

Definition 4.1. A function $\Psi: X \rightarrow \boldsymbol{R} \cup\{\infty\}$ is said to be $A$-superharmonic if it satisfies

( i ) $\Psi$ l.s.c.

(ii) $\Psi(x) \geqq \int_{X} \Psi d \mu_{x}$, all $x \in X$ and $\mu_{x} \in M_{x}^{+}(X)$. 
Definition 4.2. Let $F$ be a compact subset of $X$. $F$ has the almost norm preserving extension property, if for each $\varepsilon>0$ and $\left.a_{0} \in A\right|_{F}$ there exists a function $a \in A$ such that

$$
\left.a\right|_{F}=a_{0}, \quad\|a\|_{X} \leqq\left\|a_{0}\right\|_{F}+\varepsilon .
$$

If $\varepsilon$ can be taken to be zero in (4.1), then $F$ has the norm preserving extension property.

We shall need a criterion for the almost norm preserving extension property, which is due to Gamelin [9, p. 281] and Glicksberg [10, p. 420] (cf. also Curtis [8]). For the sake of completeness we present a short proof.

Lemma 4.3. A closed subset $F$ of $X$ has the almost norm preserving extension property if for each $\sigma \in A^{\perp}$ :

$$
\inf _{\nu \in\left(\left.A\right|_{F}\right)^{\perp}}\left\|\left.\sigma\right|_{F}+\nu\right\| \leqq\left\|\left.\sigma\right|_{X \backslash F}\right\| \text {. }
$$

Proof. The almost norm preserving extension property is tantamaunt to the equality of the uniform norm on $\left.A\right|_{F}$ and the extension norm:

$$
\left\|a_{0}\right\|_{\text {ext. }}=\inf \left\{\|a\|_{X}|a \in A, a|_{F}=a_{0}\right\} .
$$

In this norm $\left.A\right|_{F}$ is isometrically isomorphic to the quotient space $A / F^{\perp}$ where $F^{\perp}=\{a \in A \mid a \equiv 0$ on $F\}$; and we are to prove that the canonical imbedding $\rho: A /\left.F^{\perp} \rightarrow A\right|_{F}$ is an isometry from the quotient norm to the uniform norm. By duality (i.e., by Hahn-Banach) we may as well prove that the transposed map $\rho^{*}$ is an isometry. Representing the occuring functionals by measures, we can translate this statement into

$$
\inf _{\sigma \in A^{\perp}}\|\mu+\sigma\|=\inf _{\nu \in(A \mid F)^{\perp}}\|\mu+\nu\|, \text { all } \mu \in M(F) .
$$

To prove that (4.2) implies (4.5), we consider measures $\mu \in M(F)$, $\sigma \in A^{\perp}$ and an arbitrary $\varepsilon>0$. Also we can choose $\nu_{0} \in\left(\left.A\right|_{F}\right)^{\perp}$ such that

$$
\left\|\left.\sigma\right|_{F}-\nu_{0}\right\| \leqq \inf _{\nu \in(d \mid F)^{\perp}}\left\|\left.\sigma\right|_{F}-\nu\right\|+\varepsilon \leqq\|\sigma \mid X \backslash F\|+\varepsilon .
$$

Then

$$
\begin{aligned}
\|\mu-\sigma\|= & \left\|\mu-\left.\sigma\right|_{F}\right\|+\left\|\left.\sigma\right|_{X \backslash F}\right\| \geqq\left\|\mu-\nu_{0}\right\|-\left\|\nu_{0}-\left.\sigma\right|_{F}\right\| \\
& +\left\|\left.\sigma\right|_{X \backslash F}\right\| \geqq\left\|\mu-\nu_{0}\right\|-\varepsilon \geqq \inf _{\nu \in\left(\left.A\right|_{F} \perp\right.}\|\mu-\nu\| \mid-\varepsilon,
\end{aligned}
$$

which completes the proof. 
We remark for later purposes that for $\mu \in M(F)$ :

$$
\sup \left\{\left|\int_{F} a_{0} d \mu\right|\|\| a_{0} \|_{F} \leqq 1,\left.a_{0} \in A\right|_{F}\right\}=\inf _{\nu \in(A \mid F)^{\perp}}\|\mu-\nu\| .
$$

Proposition 4.4. If $F$ is a compact subset of $\partial_{A} X$ satisfying (A.1), then $F$ has the almost norm preserving extension property.

Proof. By Lemma 4.3 and the above remark (4.4), it suffices to prove that for every $\sigma \in A^{\perp}$ :

$$
\sup \left\{\left|\int_{F} a_{0} d \sigma\right|\|\| a_{0} \|_{F} \leqq 1,\left.a_{0} \in A\right|_{F}\right\} \leqq\left\|\left.\sigma\right|_{X \backslash F}\right\| \text {. }
$$

Let $\sigma \in A^{\perp}$, and $\left.a_{0} \in A\right|_{F}$, with $\left\|a_{0}\right\|_{F} \leqq 1$. Applying Lemma 3.1 we obtain

$$
0=\sigma\left(\bar{a}_{0}\right)=\int_{F} a_{0} d \sigma+\int_{F \backslash X} \bar{a}_{0} d \sigma
$$

such that

$$
\left|\int_{F} a_{0} d \sigma\right|=\left|\int_{X \backslash F} \bar{a}_{0} d \sigma\right| \leqq\left\|\left.\sigma\right|_{X \backslash F}\right\|,
$$

which completes the proof.

If $F$ is a compact subset of $\partial_{A} X$ satisfying (A.1), then $\left.A\right|_{F}$ is a closed subspace of $\mathscr{C}_{C}(F)$. In fact, $\left.A\right|_{F}$ is isometrically isomorphic to $A / F^{\perp}$.

We are now able to state and prove the main theorem. The proof of this theorem is essentially based upon Theorem 2.1 and the technique developed by Bai Andersen [3].

THEOREM 4.5. Let $F$ be a compact subset of $\partial_{A} X$ satisfying (A.1), i.e.

$$
\left.\mu \in M\left(\partial_{A} X\right) \cap A^{\perp} \Rightarrow \mu\right|_{F} \in A^{\perp} .
$$

Let $\left.a_{0} \in A\right|_{F}$ and let $\psi$ be a strictly positive A-superharmonic function on $X$ such that $\left|a_{0}(x)\right| \leqq \psi(x)$ for all $x \in F$. Then there exists $a$ function $a \in A$ such that

(i) $\left.a\right|_{F}=a_{0}$,

(ii) $|a(x)| \leqq \psi(x)$ all $x \in X$.

Proof. Without loss of generality we may assume $\psi$ to be bounded. Since $F$ satisfies the requirement (A.1), $\left.A\right|_{F}$ is closed and $\bar{a}_{0} \in \mathscr{A}$.

Thus by Theorem 2.2 we can extend $a_{0}$ to a function $a_{0}^{\prime} \in A$ such that $\left|a_{0}^{\prime}(x)\right|<\varphi(x)$ for all $x \in X$, whenever $\varphi$ is a bounded l.s.c. func- 
tion on $X$ such that $\left|\bar{a}_{0}(x)\right|<\varphi(x)$ for all $x \in X$.

Applying this to the function $\varphi_{1}=2 \psi$, we can extend $a_{0}$ to a function $a_{1} \in A$ such that $\left|a_{1}(x)\right|<2 \psi(x)$ for all $x \in X$.

Now define

$$
\varphi_{2}=2 \psi \wedge\left[2^{2}\left(\psi-2^{-1}\left|a_{1}\right|\right)\right] .
$$

The function $\varphi_{2}$ is strictly positive on all of $X$. For $x \in F$ we have $\varphi_{2}(x)=2 \psi(x)$, and hence for an arbitrary $x \in X$ :

$$
\begin{aligned}
\left|\bar{a}_{0}(x)\right| & =\left|\int_{F} a_{0} d \mu_{x}\right| \leqq \int_{F}\left|a_{0}\right| d \mu_{x}<\int_{X} 2^{2}\left(\psi-2^{-1}\left|a_{1}\right|\right) d \mu_{x} \\
& =2^{2}\left(\int_{X} \psi d \mu_{x}-2^{-1} \int_{X}\left|a_{1}\right| d \mu_{x}\right) \leqq 2^{2}\left(\psi(x)-2^{-1}\left|\int_{X} a_{1} d \mu_{x}\right|\right) \\
& =2^{2}\left(\psi(x)-2^{-1}\left|a_{1}(x)\right|\right) .
\end{aligned}
$$

Hence $\left|\bar{a}_{0}(x)\right|<\varphi_{2}(x)$ all $x \in X$.

By Theorem 2.2 we can choose $a_{2} \in A$ such that

$$
\left|a_{2}\right|<\varphi_{2},\left.\quad a_{2}\right|_{F}=a_{0} \text {. }
$$

Assume for induction that extensions $a_{1}, \cdots, a_{n} \in A$ have been constructed such that

$$
\left|a_{p}\right|<2 \psi \wedge\left[2^{p}\left(\psi-\sum_{r=1}^{p-1} 2^{-r}\left|a_{r}\right|\right)\right]=\varphi_{p}, p=2, \cdots, n,
$$

and define

$$
\varphi_{n+1}=2 \psi \wedge\left[2^{n+1}\left(\psi-\sum_{r-1}^{n} 2^{-r}\left|a_{r}\right|\right)\right] .
$$

The function $\varphi_{n+1}$ is strictly positive by induction hypothesis. For $x \in F$ we shall have

$$
2^{n+1}\left(\psi(x)-\sum_{r=1}^{n} 2^{-r}\left|a_{0}(x)\right|\right) \geqq 2^{n+1}\left(\psi(x)-\sum_{r=1}^{n} 2^{-r} \psi(x)\right)=2 \psi(x)
$$

such that $\varphi_{n+1}(x)=2 \psi(x)$. Hence for an arbitrary $x \in X$ :

$$
\begin{aligned}
\left|\bar{a}_{0}(x)\right| & =\left|\int_{F} a_{0} d \mu_{x}\right| \leqq \int_{F} \psi d \mu_{x}<\int_{X} 2^{n+1}\left(\psi-\sum_{r=1}^{n} 2^{-r}\left|a_{r}\right|\right) d \mu_{x} \\
& \leqq 2^{n+1}\left(\int_{X} \psi d \mu_{x}-\sum_{r=1}^{n} 2^{-r}\left|\int_{X} a_{r} d \mu_{x}\right|\right) \\
& \leqq 2^{n+1}\left(\psi(x)-\sum_{r=1}^{n} 2^{-r}\left|a_{r}(x)\right|\right) .
\end{aligned}
$$

Hence $\left|\bar{a}_{0}(x)\right|<\varphi_{n+1}(x)$ for all $x \in X$. Again by Theorem 2.1 we can choose $a_{n+1} \in A$ such that

$$
\left|a_{n+1}\right|<\varphi_{n+1},\left.\quad a_{n+1}\right|_{F}=a_{0} .
$$


Continuing in this way we obtain a sequence $\left\{a_{n}\right\}_{n=1}^{\infty} \subseteq A$ such that for $n=1,2, \cdots$

(i) $\left.\quad a_{n}\right|_{F}=a_{0}$,

(ii) $\psi(x)-\sum_{r=1}^{n} 2^{-r}\left|a_{r}(x)\right|>0$, all $x \in X$,

(iii) $\left\|a_{n}\right\| \leqq 2 \sup _{x \in X} \psi(x)$.

By (iii) the sequence $\sum_{r=1}^{\infty} 2^{-r} a_{r}$ is uniformly convergent and $a=$ $\sum_{r=1}^{\infty} 2^{-r} a_{r} \in A$. Clearly $\left.a\right|_{F}=a_{0}$ and it follows from (ii) that $|a(x)| \leqq$ $\psi(x)$ for all $x \in X$. This completes the proof.

Taking $\psi \equiv 1$ in Theorem 4.5 we obtain the following:

COROLlary 4.6. Let $F$ be a compact subset of $\partial_{A} X$ satisfying (A.1), i.e.

$$
\left.\mu \in M\left(\partial_{A} X\right) \cap A^{\perp} \Rightarrow \mu\right|_{F} \in A^{\perp},
$$

then $F$ has the norm preserving extension property.

REMARK. In the proof of Theorem 4.5 we have actually proved slightly more than was stated. The $A$-superharmonicity of the function $\psi$ was used just once, namely in the verification that $\left|\bar{a}_{0}(x)\right|<$ $\varphi_{n+1}(x)$ for $n=1,2, \cdots$ and all $x \in X$. However if $x$ is a point of $X$ such that

$$
\mu \in M_{x}^{+}\left(\partial_{A} X\right) \Rightarrow \mu_{x}(F)=0,
$$

then by definition $\bar{a}_{0}(x)=0$, and there is nothing to verify.

Hence, Theorem 4.5 subsists if $\psi: X \rightarrow \boldsymbol{R}^{+} \cup\{\infty\}$ is allowed to be a l.s.c. function such that

$$
\psi(x) \geqq \int \psi d \mu_{x},
$$

for all points $x \in X$ for which $\mu_{x}(F) \neq 0$ for some $\mu_{x} \in M_{x}^{+}\left(\partial_{A} X\right)$.

5. A peak set theorem. In this section we shall deal with compact subsets $F$ of $\partial_{A} X$ satisfying the requirement (A.2). For such an $F$ we define the function $\bar{\chi}_{F}$ as in (3.7).

Proposition 5.1. If $F$ is a compact subset of $\partial_{A} X$ satisfying (A.2), then the A-convex hull of $F$ is equal to the set of all $x \in X$ such that $\bar{\chi}_{F}(x)=1$.

Proof. By definition, the $A$-convex hull of $F$ is the set

$$
F^{\wedge}=\left\{x \in X|| a(x) \mid \leqq\|a\|_{F}, \text { all } a \in A\right\} \text {. }
$$

We first assume that $\bar{\chi}_{F}(x)=1$ i.e., $\mu_{x}(F)=1$ for $\mu_{x} \in M_{x}^{+}\left(\partial_{A} X\right)$. Then we obtain for every $a \in A$, 


$$
|a(x)|=\left|\int_{Y} a d \mu_{x}\right| \leqq \int_{F}|a| d \mu_{x} \leqq\|a\|_{F}
$$

such that $x \in F^{\wedge}$.

Next assume that $\bar{\chi}_{F}(x)<1$. This implies that $\Phi(x) \in S_{F}$. Hence we can separate $\Phi(x)$ and $S_{F^{\prime}}$ by a $w^{*}$-continuous linear functional on $A^{*}$ i.e., there exists a function $a \in A$ and an $\alpha \in \boldsymbol{R}$ such that

$$
\operatorname{Re} \Psi(\alpha)(\Phi(x))>\alpha>\operatorname{Re} \Psi(\alpha)\left(S_{k^{\prime}}\right) \geqq 0,
$$

and hence again

$$
\operatorname{Re} a(x)>\alpha>\operatorname{Re} a(F) \geqq 0 .
$$

Now, for sufficiently large $\delta \in \boldsymbol{R}^{+}$, the function $a+\delta \in A$ satisfies

$$
|a(x)+\delta|>\delta+\alpha>|a(y)+\delta| \text { all } y \in F .
$$

In fact, it suffices to take

$$
\delta>\frac{\gamma^{2}+\beta^{2}-\alpha \beta}{2(\alpha-\beta)},
$$

where

$$
\beta=\max \{\operatorname{Re} a(y) \mid y \in F\}<\alpha, \quad \gamma=\max \{|\operatorname{Im} a(y)| \mid y \in F\} .
$$

Hence

$$
\|a+\delta\|_{F}<|a(x)+\delta|
$$

i.e., $\curvearrowright \notin F^{\wedge}$, which completes the proof.

Lemma 5.2. Let $F$ be a compact subset of $\partial_{A} X$ satisfying (A.2), for which $\left.A\right|_{F}$ is closed in $\mathscr{C}_{C}(F)$. Let is be a strictly positive $A$ superharmonic function on $X$ such that $1 \leqq \psi(x)$ for all $\rightsquigarrow \in F$.

Then there exists a function $a \in A$ such that

$$
\left.a\right|_{F}=1, \quad|a(x)| \leqq \psi(x) \text { all } x \in X
$$

Proof. Since $\bar{\chi}_{F}$ is an element of $\mathscr{A}$ and $\left.A\right|_{F}$ is assumed to be closed in $\mathscr{C}_{C}(F)$ we can use Theorem 2.2 with $\left.a_{0} \in A\right|_{F}, a_{0} \equiv 1$. Now using the same technique as in the proof of Theorem 4.5 we obtain a function $a \in A$ satisfying (5.2).

Lemma 5.3. Let $F$ be a compact subset of $\partial_{A} X$ satisfying (A.2), and let $G$ be a compact subset of $X \backslash F^{\wedge}$. Then there exists an $A$ superharmonic function $\psi$ on $X$ such that:

(i) $\quad$ s $(x)=1$ for all $x \in F^{\wedge}$

(ii) $|\psi(x)|<1$ for all $x \in G$

(iii) $0<\psi(x) \leqq 1$ for all $x \in X$. 
Proof. We write $S_{G}=\overline{c o}(\Phi(G))$ and claim that $S_{F} \cap S_{G}=\varnothing$.

To prove this, we assume for contradiction that there exists a $p_{0} \in S_{F} \cap S_{G}$, and we recall that $\hat{\chi}_{S_{F}}$ is u.s.c. and affine (since $S_{F}$ is a parallel face) and that $\hat{\chi}_{S_{F}}$ is related to $\bar{\chi}_{F}$ by formula (3.8). Now we obtain

$$
1=\hat{\chi}_{S_{F}}\left(p_{0}\right)=\max _{p \in S_{G}} \hat{\chi}_{S_{F}}(p)=\max _{p \in \Phi(G)} \hat{\chi}_{S_{F}}(p)=\max _{p \in G} \bar{\chi}_{F}(p) .
$$

By Proposition 5.1, this contradicts the hypothesis $G \cap F^{\wedge}=\varnothing$, and the claim is proved.

Now there exists a number $\delta$ such that

$$
\max _{p \in S_{G}} \hat{\chi}_{S_{F}}(p)<\delta<1,
$$

and hence we can define two disjoint convex subsets of $A^{*} \times \boldsymbol{R}$ by the formulas:

$$
\begin{aligned}
& F_{0}=\left\{(p, \alpha) \mid p \in S, \alpha \in \boldsymbol{R}, 0 \leqq \alpha \leqq \hat{\chi}_{S_{F}}(p)\right\} \\
& F_{1}=\left\{(p, \alpha) \mid p \in S_{G}, \alpha \in \boldsymbol{R}, \delta \leqq \alpha\right\}
\end{aligned}
$$

The set $F_{0}$ is compact and the set $F_{1}$ is closed. Hence we can use Hahn-Banach separation to obtain a function $b \in A$ such that

and

$$
\hat{\chi}_{s_{F}}(p)<\operatorname{Re} \psi(b)(p), \text { all } p \in G,
$$

$$
\operatorname{Re} \psi(b)(p)<\delta<1, \text { all } p \in S_{G} \text {. }
$$

The function $\psi=\operatorname{Re}(b) \wedge 1$ is $A$-superharmonic and satisfies (i), (ii) and (iii).

THeOREM 5.4. Let $X$ be a metrizable compact Hausdorff space and let $F$ be a compact subset of $\partial_{A} X$ which satisfies (A.2) i.e.

$$
\mu \in M\left(\partial_{A} X\right) \cap A^{\perp} \Rightarrow \mu(F)=0,
$$

and for which $\left.A\right|_{F}$ is closed. Then there exists a function $a \in A$ such that

$$
\left.a\right|_{F^{\wedge}}=1, \quad|a(x)|<1 \text { all } x \in X \backslash F^{\wedge},
$$

i.e. the A-convex hull of $F$ is a peak set.

Proof. By metrizability $F^{\wedge}$ is a $G_{\delta}$-set, and we can write $X \backslash F^{\wedge}=$ $\bigcup_{u=1}^{\infty} K_{n}$, where $K_{n}$ is closed.

Now we use Lemma 5.3 to obtain strictly positive $A$-superharmonic functions $\psi_{n}$ on $X$ such that $\psi_{n}(x)=1$ for all $x \in F^{\wedge}, \psi_{n}(x)<1$ for all $x \in K_{n}, \quad n=1,2, \cdots$ 
and $\psi_{n}(x) \leqq 1$ for all $x \in X$. It follows from Lemma 5.2 that there exist functions $a_{n} \in A$ such that $\left.a_{n}\right|_{F}=1$ and $\left|a_{n}(x)\right| \leqq \psi_{n}(x)$ for all $x \in X$. Now the function

$$
a=\sum_{n=1}^{\infty} 2^{-n} a_{n}
$$

satisfies (5.5) and the proof is complete.

REMARK. Actually the conclusion of Theorem 5.4 subsists under more general assumptions. The metrizability of $X$ was only invoked to make $F^{\wedge}$ a $G_{\delta}$-set. In particular we shall have the following:

COROLlary 5.5. Let $x \in \partial_{A} X$ be a $G_{\tilde{\delta}}$-point satisfying (A.2), i.e.

$$
\mu \in M\left(\partial_{A} X\right) \cap A^{\perp} \Rightarrow \mu(\{x\})=0,
$$

then $x$ is a peak point for $A$.

Finally we remark that if $X$ is a metrizable compact Hausdorff space and $F$ is a compact subset of $\partial_{A} X$ satisfying the stronger condition (A.1) then the A-convex hull of $F$ is a peak set.

6. Relations to the Bishop-Rudin-Carleson Theorem. In the present chapter we shall consider a compact subset $F$ of $X$ satisfying the requirement

$$
\left.\mu \in A^{\perp} \Longrightarrow \mu\right|_{F^{\prime}}=0 \text {. }
$$

Clearly (B) is more restrictive than (A.1), and a fortiori than (A.2). Note also that (B) implies $F^{\prime} \subset \partial_{\alpha} X$ since $M_{x}^{+}(X)=\left\{\varepsilon_{x}\right\}$ for all $x \in F$.

If $x \notin F$ and $\mu_{x} \in M_{x}^{+}(X)$, then $\varepsilon_{x}-\mu_{x} \in A^{\perp}$. Now the requirement (B) implies $\left.\left(\varepsilon_{x}-\mu_{x}\right)\right|_{F}=0$, such that $\mu_{x}(F)=0$. By the definition (3.2) we shall have $\bar{a}_{0}(x)=0$. Hence

$$
\bar{a}_{0}=a_{0} \cdot \chi_{F^{\prime}} \cdot
$$

Transferring to the state space and making use of (3.8), we observe that the function $\hat{\chi}_{S_{F}}$ takes the value zero on $\Phi(X \backslash F)$. Geometrically, this means that the canonical embedding $\Phi: X \rightarrow S$ maps $F$ into the (compact) split face $S_{F}=\overline{c o}(\Phi(F))$, and $X \backslash F$ into the complementary $\left(G_{\hat{o}^{-}}\right)$face $S_{F}^{\prime}(c f \cdot$ [2, Cor. 1.2]).

It follows from (6.1) that $\bar{\chi}_{F}=\chi_{F}$ and by Proposition 5.1 we obtain $F=\hat{F}$. Moreover, it follows from Proposition 4.4 that $\left.A\right|_{F}$ is a closed subspace of $\mathscr{C}_{C}(F)$, and it follows from (B) that $\left(\left.A\right|_{F}\right)^{\perp}=(0)$. Hence $\left.A\right|_{F}=\mathscr{C}_{C}(F)$. Also it follows from the results of chapter 5 that if $F$ is a $G_{\hat{o}}$, then it is a peak set. 
In other words: If $F$ satisfies (B) then it is an interpolation set; and if in addition it is a $G_{o}$, then it is a peak-interpolation set.

Finally we note that we may apply Theorem 4.5 in the form stated in the Remark at the end of $\S 4$, to obtain:

THEOREM 6.1. (Bishop-Rudin-Carleson) Let $F$ be a compact subset of $X$ satisfying (B), i.e.

$$
\left.\mu \in A^{\perp} \Rightarrow \mu\right|_{F}=0
$$

let $f_{0} \in \mathscr{C}_{\boldsymbol{C}}(F)$, and let $\psi: X \rightarrow \boldsymbol{R}^{+} \cup\{\infty\}$ be a strictly positive 1.s.c. function such that $\left|f_{0}(x)\right| \leqq \psi(x)$ for all $x \in F$. Then there exists an $a \in A$ such that $\left.a\right|_{F}=f_{0}$ and $|a(x)| \leqq \psi(x)$ for all $x \in X$.

REMARK. Theorem 6.1 is the most general form of the BishopRudin-Carleson Theorem. Originally Bishop stated and proved this theorem for a continuous function $\psi$ and strict inequality sign [4]. Appealing to the inductive construction of Pełczynski [12], Semadeni improved it to the form stated above [16]. (Cf. also Michael-Pełczynski $[11$, p. 569]).

7. The sup-norm algebra case. In this section we shall assume that $A$ is a sup-norm algebra, and we shall consider two new requirements on a compact subset $F$ of $\partial_{A} X$ :

$$
\begin{aligned}
& \left.\mu \in A^{\perp} \Rightarrow \mu\right|_{F} \in A^{\perp} \\
& \left.\mu \in A^{\perp} \Rightarrow \mu\right|_{F} \wedge \in A^{\perp} .
\end{aligned}
$$

Clearly (B) implies (G.1) and (G.2), and each one of these implies (A.1). In fact, (G.2) implies (A.1) since $\left.\mu\right|_{F^{\wedge}}=\left.\mu\right|_{F}$ for every $\mu \in M\left(\partial_{A} X\right)$. This result in turn is elementary, but not entirely obvious, so we shall sketch a short proof: Note first that $F^{\wedge}=\Phi^{-1}\left(S_{F}\right)$, so that $F$ can be thought of as the intersection of $X$ with the ordinary closed convex hull of $F$ in $S$. (This is standard for real function spaces, and the complex case is taken care of by the same argument as in the proof of Proposition 5.1.). Hence the problem is reduced to show the general implication:

$$
\operatorname{Supp}(\nu) \subset \overline{c o}(Q) \Rightarrow \operatorname{Supp}(\nu) \subset Q,
$$

where $\nu$ is a boundary measure and $Q$ is a closed subset of $S$. By an elementary theorem $\nu$ is also a boundary measure on $\overline{c o}(Q)$. (An explicite proof is given in [3, Lem. 1].) Hence $\nu$ is supported by the closure of the extreme points of $\overline{c o}(Q)$. By Milman's Theorem Supp $(\nu) \subset Q$, and the implication is proved.

In [9] and [10] Gamelin and Glicksberg have dealt with the requirement (G.1), and from their works we shall adopt the following: 
DEFinition 7.1. Let $F$ be a compact subset of $X$ and let $t>0$. $\left.A\right|_{F}$ is said to have the property $E_{t}$ if the following conditions holds: Given $\left.f \in A\right|_{F}$ with $\|f\|_{F}<1$ and a compact subset $G$ of $X \backslash F$, there exists an extension $g \in A$ of $f$ such that

$$
\|g\|_{X}<\max \{1, t\}, \quad|g(x)|<t \text { all } x \in G .
$$

The extension constant $e(A, F)$ of $F$ associated with $\left.A\right|_{\Gamma}$ is defined by the formula:

$$
e(A, F)=\inf \left\{t|A|_{F} \text { has property } E_{t}\right\} .
$$

If $\left.A\right|_{F}$ has property $E_{t}$ for no $t$, then we define $e(A, F)=\infty$.

The connection between the extension constant and the requirement (G.1) is expressed in the following:

THEOREM 7.2. (Gamelin-Glicksberg). Let $F$ be a compact subset of $X$. Then the following conditions are equivalent:

(i ) $\left.\mu \in A^{\perp} \Longrightarrow \mu\right|_{F} \in A^{\perp}$

(ii) $e(A, F)=0$

(iii) $F$ is an intersection of peak sets for $A$.

Proof. See [9] and [10].

Proposition 7.3. Let $A$ be a sup-norm algebra over $X$ and let $F$ be a compact subset of $\partial_{A} X$ satisfying the requirement (A.1). Also let $G$ be a compact subset of $X \backslash F^{\wedge}$ and let $\varepsilon>0$. Then there exists a function $a \in A$ such that

(i ) $a(x)=1$ for all $x \in F^{\wedge}$

(ii) $|a(x)|<\varepsilon$ for all $x \in G$

(iii) $\|a\|_{x}=1$.

Proof. Choose $\psi$ as in Lemma 5.3 and let $\left.a_{0} \in A\right|_{F}, a_{0} \equiv 1$. Using. Theorem 4.5 we obtain a function $b \in A$ such that

$$
\left.b\right|_{F}=1, \quad|b(x)| \leqq \psi(x) \text { for all } x \in X .
$$

Cleary $b(x)=1$ for all $x \in F^{\wedge}$ and $|b(x)|<1$ for all $x \in G$. Now choose a natural number $n$ such that $\left(\|b\|_{G}\right)^{n}<\varepsilon$ and define $a=b^{n}$. The proof is complete.

We are now able to clarify the connection between (A.1) and the extension constant of $F^{\wedge}$.

Theorem 7.4. Let $A$ be a sup-norm algebra over $X$ and let $F$ be a compact subset of $\partial_{A} X$. Then $e\left(A, F^{\wedge}\right)=0$ if and only if $F$ satisfies (A.1) i.e. 


$$
\left.\mu \in M\left(\partial_{A} X\right) \cap A^{\perp} \Rightarrow \mu\right|_{F} \in A^{\perp}
$$

Proof. By virtue of Theorem 7.2 and the fact that $\left.\mu\right|_{F^{\wedge}}=\left.\mu\right|_{F}$ for every $\mu \in M\left(\partial_{A} X\right)$, if follows that $e\left(A, F^{\wedge}\right)=0$ implies (A.1).

Now assume (A.1) and let $\left.a_{0} \in A\right|_{F \wedge}$ with $\left\|a_{0}\right\|_{F^{\wedge}}=\left\|a_{0}\right\|_{F}<1$. Let $G$ be a compact subset of $X \backslash F^{\wedge}$ and let $\varepsilon>0$. We choose $b \in A$ such that $\|b\|_{X}=\left\|a_{0}\right\|_{F}$ and $\left.b\right|_{F}=\left.a_{0}\right|_{F}$ according to Corollary (4.6), and we choose $h \in A$ according to Proposition (7.3) i.e.

$$
\left.h\right|_{F^{\wedge}}=1, \quad|h(x)|<\varepsilon \text { for all } x \in G
$$

and $\|h\|_{X}=1$. Then we define $a=h \cdot b \in A$. Now, $a$ is a norm preserving extension of $a_{0}$ and $|a(x)|<\varepsilon$ for all $x \in G$. Hence $\left.A\right|_{F^{\wedge}}$ has property $E_{\varepsilon}$ for all $\varepsilon>0$, and so we have proved that $e\left(A, F^{\wedge}\right)=0$.

Thus we see how the requirements (A.1), (G.1) and (G.2) are related for sup norm algebras. (A.1) and (G.2) are always equivalent for every compact subset $F$ of $\partial_{A} X$, and if in addition $F$ is $A$-convex, then they are equivalent to (G.1). This is not always the case even if $A$ is an algebra and $F$ satisfies (A.1), as can be seen from the following example

\section{Example 7.5. (The "Tomato Can Algebra").}

Let $X \subset \boldsymbol{R} \times \boldsymbol{C}$ be defined as $\{(t, z)|t \in[0,1]| z \mid, \leqq 1\}$; let $A$ be the sup-norm algebra consisting all functions $f \in \mathscr{C}_{C}(X)$ such that $f(0, z)$ is analytic for $|z|<1$; and let $F=\{(0, z)|| z \mid=1\}$. Then $F$ satisfies (A.1) and $F^{\wedge}=\{(0, z)|| z \mid \leqq 1\}$.

\section{Proof. We first note that}

$$
\left.\left.\partial_{A} X=\{(t, z) \mid t \in] 0,1\right],|z| \leqq 1 \text { or } t=0,|z|=1\right\} .
$$

Hence the Shilov boundary $\partial_{S} A=\overline{\partial_{A} X}$ is all of $X$, and it also follows that $X$ is the maximal ideal space $M_{A}$ of $A$.

If $G$ is a compact subset of $X \backslash\{(0, z)|| z \mid \leqq 1\}$, then $G$ is a peak interpolation set for $A$ and $\left.A\right|_{G}=\mathscr{C}_{C}(G)$. Hence if $\mu \in A^{\perp}$ then $\left.\mu\right|_{G}=0$. In other words $\operatorname{supp}(\mu) \subset\{0, z)|| z \mid \leqq 1\}$ for all $\mu \in A^{\perp}$.

Now assume $\mu \in M\left(\partial_{A} X\right) \cap A^{\perp}$. Then $\left.\mu\right|_{F}=\mu \in A^{\perp}$. Hence $F$ satisfies (A.1) but trivially $F^{\wedge}=\{(0, z)|| z \mid \leqq 1\}$; and the proof is complete.

This example shows also that (A.1) and (G.1) need not be equivalent even if we consider $A$ as a sup-norm algebra over the maximal ideal space or the Shilov boundary.

Finally we remark that if $X$ is a compact subset of $C$ and 
$A=\left.R(X)\right|_{\partial X}$, i.e., if $A$ is the uniform closure of the rational functions on $X$ considered as a function algebra over the topological boundary $\partial X$, then the two conditions (A.1) and (G.1) are equivalent since $F=F^{\wedge}$ for every compact subset $F$ of $\partial_{A} X$. In fact for a point $z_{0} \in \partial X \backslash F$ we choose $f=\left(1 / z-z_{1}\right) \in A$, where $z_{1} \in X$ and

$$
\left|z_{1}-z_{0}\right|=\frac{1}{2} \inf \left|z-z_{0}\right|,
$$

and obtain $\left|f\left(z_{0}\right)\right|=2 \sup |f(z)|$.

\section{REFERENCES}

1. E. M. Alfsen, Compact convex sets and boundary integrals, Ergebnisse der Matematik 57, Springer Verlag, Germany.

2. E. M. Alfsen and T. B. Andersen, Split faces of compact convex sets, to appear in Proc. London Math. Soc. 21 (1970), 415-442.

3. T. B. Andersen, On dominated extensions of continuous affine functions on split faces, (to appear).

4. Bishop, A general Rudin-Carleson theorem, Proc. Amer. Math. Soc., 13 (1962), 140-143.

5. J. E. Björk, Private communication.

6. - Interpolation on closed linear subspaces of $C(X)$, (to appear).

7. E. Brièm, Restrictions of subspaces of $C(X)$, Investigationes Math. 10 (1970), 288297.

8. P. C. Curtis, Topics in function spaces, Lecture notes, Univ. of Århus 1969/70.

9. T. W. Gamelin, Restrictions of subspaces of $C(X)$, Trans. Amer. Math. Soc., 112 (1964), 278-286.

10. I. Glicksberg, Measures orthogonal to algebras and sets of antisymmetry, Trans. Amer. Math. Soc. 105 (1962), 415-435.

11. E. Michael and A. Pelczynski, A linear extension theorem, Illinois Journal of Mathematics, 11 (1967), 563-579.

12. A. Pelczynski, Supplement to my paper "On simultaneous extensions of continuous functions, Studia. Math. 25 (1964), 157-161.

13. R. R. Phelps, Lectures on Choquet's theorem, Van Nostrand, New York, 1966.

14. M. Rao, Measurable selections of representing measures, Århus Univ. Math. Inst. Preprint Ser. 1969/70 No. 24.

15. M. Rogalski, Topologies faciales dans les convexes compacts; calcul functionel et décomposition spectrale dans le centre d'un espace $A(X)$, Seminaire Choquet 1969-70 No. 4.

16. Z. Semadeni, Simultaneous extensions and projections in spaces of continuous functions, Lecture Notes, Univ. of Århus 1965.

Received June 18, 1970.

UNiversity of OSLO, Norway

AND

UNIVERsity of Århus. DenMark 


\title{
PACIFIC JOURNAL OF MATHEMATICS
}

\section{EDITORS}

H. SAMELSON

Stanford University

Stanford, California 94305

C. R. HobвY

University of Washington

Seattle, Washington 98105

\section{J. DugunduI}

Department of Mathematics

University of Southern California

Los Angeles, California 90007

RICHARD ARENS

University of California

Los Angeles, California 90024

\section{ASSOCIATE EDITORS}
E. F. BECKENBACH
B. H. Neumann
F. WOLE
K. Yoshida

\section{SUPPORTING INSTITUTIONS}

\author{
UNIVERSITY OF BRITISH COLUMBIA \\ CALIFORNIA INSTITUTE OF TECHNOLOGY \\ UNIVERSITY OF CALIFORNIA \\ MONTANA STATE UNIVERSITY \\ UNIVERSITY OF NEVADA \\ NEW MEXICO STATE UNIVERSITY \\ OREGON STATE UNIVERSITY \\ UNIVERSITY OF OREGON \\ OSAKA UNIVERSITY \\ UNIVERSITY OF SOUTHERN CALIFORNIA
}

\author{
STANFORD UNIVERSITY \\ UNIVERSITY OF TOKYO \\ UNIVERSITY OF UTAH \\ WASHINGTON STATE UNIVERSITY \\ UNIVERSITY OF WASHINGTON

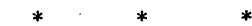 \\ AMERICAN MATHEMATICAL SOCIETY \\ CHEVRON RESEARCH CORPORATION \\ NAVAL WEAPONS CENTER
}

The Supporting Institutions listed above contribute to the cost of publication of this Journal, but they are not owners or publishers and have no responsibility for its content or policies.

Mathematical papers intended for publication in the Pacific Journal of Mathematics should be in typed form or offset-reproduced, (not dittoed), double spaced with large margins. Underline Greek letters in red, German in green, and script in blue. The first paragraph or two must be capable of being used separately as a synopsis of the entire paper. The editorial "we" must not be used in the synopsis, and items of the bibliography should not be cited there unless absolutely necessary, in which case they must be identified by author and Journal, rather than by item number. Manuscripts, in duplicate if possible, may be sent to any one of the four editors. Please classify according to the scheme of Math. Rev. Index to Vol. 39. All other communications to the editors should be addressed to the managing editor, Richard Arens, University of California, Los Angeles, California, 90024.

50 reprints are provided free for each article; additional copies may be obtained at cost in multiples of 50 .

The Pacific Journal of Mathematics is published monthly. Effective with Volume 16 the price per volume (3 numbers) is $\$ 8.00$; single issues, $\$ 3.00$. Special price for current issues to individual faculty members of supporting institutions and to individual members of the American Mathematical Society: $\$ 4.00$ per volume; single issues $\$ 1.50$. Back numbers are available.

Subscriptions, orders for back numbers, and changes of address should be sent to Pacific Journal of Mathematics, 103 Highland Boulevard, Berkeley, California, 94708.

PUBLISHED BY PACIFIC JOURNAL OF MATHEMATICS, A NON-PROFIT CORPORATION

Printed at Kokusai Bunken Insatsusha (International Academic Printing Co., Ltd.), 7-17, Fujimi 2-chome, Chiyoda-ku, Tokyo, Japan. 


\section{Pacific Journal of Mathematics}

\section{Vol. 36, No. $3 \quad$ BadMonth, 1971}

E. M. Alfsen and B. Hirsberg, On dominated extensions in linear subspaces of

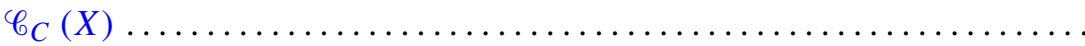

Joby Milo Anthony, Topologies for quotient fields of commutative integral

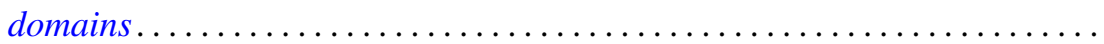

V. Balakrishnan, G. Sankaranarayanan and C. Suyambulingom, Ordered cycle lengths in a random permutation .......................... 603

Victor Allen Belfi, Nontangential homotopy equivalences............... 615

Jane Maxwell Day, Compact semigroups with square roots .............. 623

Norman Henry Eggert, Jr., Quasi regular groups of finite commutative nilpotent algebras .......................................... 631

Paul Erdős and Ernst Gabor Straus, Some number theoretic results ......... 635

George Rudolph Gordh, Jr., Monotone decompositions of irreducible Hausdorff continua .............................................

Darald Joe Hartfiel, The matrix equation $A X B=X \ldots \ldots \ldots \ldots \ldots \ldots . \ldots 69$

James Howard Hedlund, Expansive automorphisms of Banach spaces. II . . . . 671

I. Martin (Irving) Isaacs, The p-parts of character degrees in p-solvable

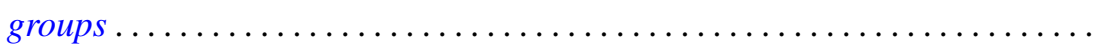

Donald Glen Johnson, Rings of quotients of $\Phi$-algebras ............... 693

Norman Lloyd Johnson, Transition planes constructed from semifield planes....

Anne Bramble Searle Koehler, Quasi-projective and quasi-injective modules.

James J. Kuzmanovich, Completions of Dedekind prime rings as second endomorphism rings...

B. T. Y. Kwee, On generalized translated quasi-Cesàro summability ...

Yves A. Lequain, Differential simplicity and complete integral closure

741

Mordechai Lewin, On nonnegative matrices.

753

Kevin Mor McCrimmon, Speciality of quadratic Jordan algebras ...

Hussain Sayid Nur, Singular perturbations of differential equations in abstract spaces .....................................

D. K. Oates, A non-compact Krein-Milman theorem .

Lavon Barry Page, Operators that commute with a unilateral shift on an invariant subspace...

Helga Schirmer, Properties of fixed point sets on dendrites.

Saharon Shelah, On the number of non-almost isomorphic models of $T$ in a

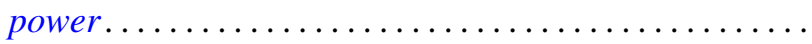

Robert Moffatt Stephenson Jr., Minimal first countable Hausdorff spaces....

Masamichi Takesaki, The quotient algebra of a finite von Neumann algebra 